\title{
RELATIONSHIP OF CLINICOPATHOLOGICAL PARAMETERS AND PREOPERATIVE CA19-9 LEVEL IN PATIENTS WITH GASTRIC CANCER
}

\author{
SHIRIN L ${ }^{1}$, RAHMAN MM ${ }^{2}$, AHMED $^{3}$, HOSSAIN MN ${ }^{4}$
}

\begin{abstract}
Objective: This study is an evaluation of serum level of the tumor marker CA 19-9 in gastric cancer patients in preoperative periods to assess the relationship of level of tumor markers in serum and clinicopathological parameters in gastric cancer.

Methods: A prospective study was done of 61 patients diagnosed with gastric cancer treated at a single institution in Bangladesh National Institute of Cancer Research and Hospital (NICRH), Dhaka, from July 2010 to December 2011. Analyses were performed to identify patient and tumor-related characteristics in gastric cancer patients. The sera from 61patients with gastric cancer were measured for CA19-9 level using a commercial immunoradiometric assay. All the patients underwent diagnostic imaging with computed tomography (CT) or ultrasound (US) before laparotomy. Metastasis was confirmed by either by US or CT and direct visualization of metastatic deposits after laparotomy.

Results: The serum levels of CA 19-9 e" the cutoff value of $40 \mathrm{U} / \mathrm{ml}$ was regarded as positive. The serum levels of CA 19-9 d" the cutoff value of $40 \mathrm{U} / \mathrm{ml}$ was considered as negative. Clinicopathological factors age, sex, tumor infiltration, $N$-classification, staging and grading was compared with Ca19-9 level in gastric carcinoma. Preoperative levels of CA19-9 was above the cut-off levels in 23\% of all cases. In the present study, we found CA 19-9 positivity was not significantly related with age (p.48) \& sex (p.35) of the patients. There was no correlation with the histologic type and CA 19-9 positivity (P.19). CA 19-9 positivity had correlation with the proportions of depth of invasion (T stage) (P.007), lymph node involvement (P.000), metastasis ( $p$.03); but no correlation with stage $(P .4)$ in case of gastric carcinoma.
\end{abstract}

Key Words: Ca 19-9 level, gastric carcinoma, metastasis.

J Dhaka Med Coll. 2014; 23(1) : 60-67.

\section{Introduction}

Carcinoma of the stomach is a major cause of cancer mortality worldwide. Its prognosis tends to be poor with cure rates little better than 5$10 \%$, although better results are obtained in Japan where the disease is common. ${ }^{1}$ There are marked variations in the incidence of gastric cancer worldwide. In Japan the disease is much more common, with an incidence of approximately 70 cases per 100000 population per year, and there are small geographical areas in China where the incidence is double that in Japan. These underlying epidemiological data make it clear that this is an environmental disease. ${ }^{1}$ Incidence of gastric carcinoma as well as other gastric malignancies is increasing day by day in the whole world as well as in Bangladesh. Prevalence of Helicobacter pylori in this country is an important contributing factor in this increasing trend of gastric malignancies. ${ }^{2}$

Tumor markers may be useful for the management of patients with cancer and several such markers are associated with gastric cancer, including carcinoembryonic antigen (CEA), carbohydrate antigen (CA 19.9) and tumor-associated glycoprotein-72 (TAG-72).

1. Dr. Laila Shirin, Assistant Professor of Surgery, Comilla Medical College, Comilla.

2. Dr. Md. Mizanur Rahman, Professor of Surgical Oncology, National Institute of Cancer Research and Hospital (NICRH), Dhaka.

3. Dr. Saifuddin Ahmed, Professor of Surgical Oncology, Bangabandhu Sheikh Mujib Medical University (BSMMU), Dhaka.

4. Dr. Md. Nabir Hossain, Junior Consultant Surgery, NICRH, Dhaka

Correspondence: Dr Laila Shirin, Assistant Professor, Department of Surgery, Comilla Medical College, Comilla. 
However, these antigens are not specific for gastric cancer since elevated levels may also be found in other neoplasms, particularly gastrointestinal. ${ }^{3}$

CA19-9 is a carbohydrate antigen identified as a glycolipid-that is, sialylated lacto-Nfucopentaose II ganglioside, which is a sialylated derivative of the Lewis blood group antigen. It is synthesized by normal human pancreatic and biliary ductular cells and by gastric, colonic, endometrial, and salivary epithelia. ${ }^{4}$ Gastric cancer-specific tumor markers have not yet been identified, and the tumor markers currently in use have very little benefit as screening tests due to their low sensitivity in early gastric cancer. ${ }^{5}$ However, the carcinoembryonic antigen CEA and the carbohydrate antigen CA 19.9 are commonly used as serum markers for this neoplasm. ${ }^{6}$

Although CEA and CA 19-9 are not applied to TNM staging according to the American Joint Committee on Cancer (AJCC) $7^{\text {th }}$ edition, ${ }^{7}$ they have been recognized as prognostic factors. This study was conducted to find out the correlation of preoperative CA 19-9 level and the factors like age, sex, tumor infiltration, Nclassification, staging, and grading in gastric carcinoma.

\section{Methods:}

A prospective study was done of 61 patients diagnosed with gastric cancer treated at a single institution in Bangladesh National Institute of Cancer Research and Hospital (NICRH), Dhaka, Bangladesh from July 2010 to December 2011. Analyses were performed to identify patient and tumor-related characteristics in gastric cancer patients. The sera from 61 patients with gastric cancer were measured for CA19-9 level using a commercial immunoradiometric assay. All the patients underwent diagnostic imaging with computed tomography (CT) or ultrasound (US) before laparotomy. Metastasis was confirmed by either by US or CT and direct visualization of metastatic deposits after laparotomy.
Patients were having adenocarcinoma of the stomach diagnosed by endoscopy of upper GIT with biopsy were evaluated for treatment.

Inclusion criteria for the study was histologically diagnosed cases of gastric adenocarcinoma and exclusion criteria were patients already received operative treatment, chemotherapy and radiotherapy, patients who have no Ca19-9 level and who do not want to include in the study. Peripheral blood samples for CA19-9 were obtained from each patient before surgery. Their data were used for comparison with the patients' clinicopathological parameters in carcinoma stomach patients. Carbohydrate antigen 19-9 (CA 19-9) were measured by counting immunoassay using a Accu-Bind ELISA Microwells CA 19-9 kit (Monobind, USA). The cutoff values of CA $19-9$ was set as recommended by the manufacturers as $40 \mathrm{U} /$ $\mathrm{mL}$.

Intraoperative staging: At operation table, gastric cancers were staged for local, nodal and metastatic spread. A D1 (with left gastric) lymph node dissection was carried out in all patients undertaking curative resection.

\section{Results:}

A total of 61 patients having cancer of stomach was undertaken for the study; among them 46 patients was male $(75 \%)$ and 15 patients was female $(25 \%)$ with a male female ratio of $3: 1$ (Table I).

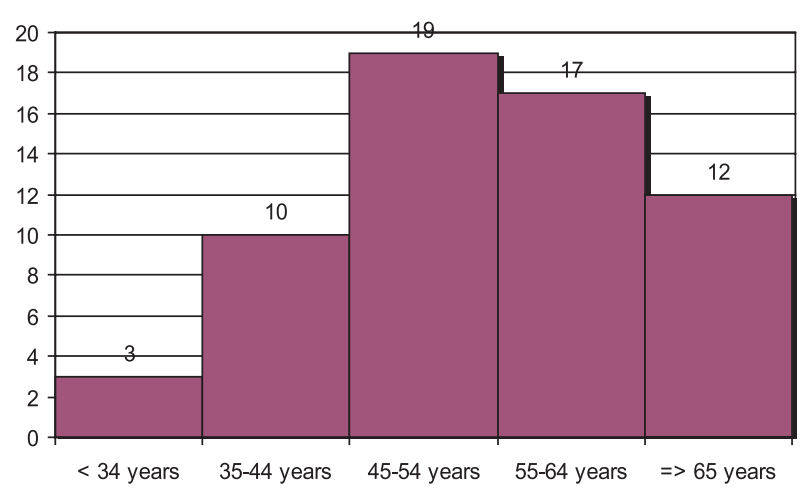

Fig. 1: Histogram showing age distribution of patients. 
Among the patients histogram showed that most of the patients age was more than 45 years, 19 patients' age was 45-54 years, 17 patients was 55-64 years and 12 patients was $>65$ years) (Fig. 1). A total of $14(23 \%)$ patients were seropositive for CA19-9 (range $=0.01$ $848.0 \mathrm{U} / \mathrm{mL}$ ). The tumor staging was completed according to AJCC classification of gastric carcinoma (Table I).

Table I

Patients' Charecterstics ( $n=61)$

\begin{tabular}{lcc}
\hline Variable & Number & $\%$ \\
\hline Age & & \\
$<55$ & 37 & 60.7 \\
$>55$ & 24 & 39.3 \\
Sex & & \\
Male & 46 & 75.4 \\
Female & 15 & 24.6 \\
CA 19-9 level & & \\
40 IU / L & 47 & 77.0 \\
$>40$ IU/L & 14 & 23.0 \\
\hline
\end{tabular}

CA 19-9 ( Cancer antigen !9-9)

Table II showed Demography of the patients with gastric cancer in 61 cases. Symptoms at presentation, were weight loss in 25 (41.0\%), vomiting in $41(67.2 \%)$. Pain on epigastrium was presented by 39 (63.9\%), anorexia by 34 $(55.7 \%)$, dysphagia by $4(6.8 \%)$ of patients. Malena was presented in $8(13.1 \%)$. Regarding haemoglobin level in $4(6.6 \%)$ cases $5-7 \mathrm{gm} / \mathrm{dl}$, in $34(55.7 \%)$ cases $7-10 \mathrm{gm} / \mathrm{dl}$ cases. Nutritional Status poor in 23 (37.7\%), average in $29(47.5 \%)$ cases. Anemia requiring blood transfusion was in 24 (39.3\%). History of smoking in this group was 37 (60.7\%). Primary tumor site was the pyloric part $26(42.6 \%)$ body and antrum in $12(19.7 \%)$ and diffuse involvement of stomach in 22(36.1\%) patients.

Table III showed that most of the patients were at stage III (29.5\%) and 31 at stage IV (50.8\%). Among differentiation moderately differentiated 28 (45.9\%) \& poorly differentiated was 24 (39.3\%). Total radical gastrectomy was performed in 5 patients, distal gastrectomy in 28 , and gastric bypass surgery in 9 patients.
Table II

Demography of the patients with gastric cancer $(n=61)$

\begin{tabular}{lcc}
\hline Variable & Value & $\%$ \\
\hline Symptoms at presentation & & \\
$\quad$ Weight loss & 25 & 41.0 \\
$\quad$ Vomiting & 41 & 67.2 \\
$\quad$ Pain on epigastrium & 39 & 63.9 \\
$\quad$ Anorexia & 34 & 55.7 \\
$\quad$ Dysphagia & 4 & 06.8 \\
$\quad$ Malena & 8 & 13.1 \\
$\quad$ Lump on abdomen & 18 & 21.3 \\
Haemoglobin level & & \\
5-7 gm/dl & 4 & 6.6 \\
7-10 gm/dl & 30 & 55.7 \\
$\quad$ 10 gm/dl & 23 & 37.7 \\
Nutritional Status & & \\
$\quad$ Poor & 23 & 37.7 \\
$\quad$ Average & 29 & 47.5 \\
$\quad$ Good & 9 & 14.8 \\
Anemia requiring transfusion & 24 & 39.3 \\
History of smoking & 37 & 60.7 \\
History of alcohol use & 2 & 3.3 \\
Primary tumor site & & \\
$\quad$ Pyloric part & 26 & 42.6 \\
$\quad$ Body and antrum & 12 & 19.7 \\
$\quad$ Fundus \& GO junction & 1 & 1.6 \\
$\quad$ Diffuse involvement & 22 & 36.1 \\
\hline
\end{tabular}

Table III

Pathological characterstics and operability of the patients in gastric cancer $(n=61)$

\begin{tabular}{lcc}
\hline Variable & Number & $\%$ \\
\hline Stage & & \\
I & 4 & 6.6 \\
II & 8 & 13.1 \\
III & 18 & 29.5 \\
IV & 31 & 50.8 \\
Resectability & & \\
Resectable & 33 & 54.1 \\
Non resectable & 18 & 29.5 \\
Not operated & 10 & 16.4 \\
Grading & & \\
Well differentiated & 9 & 14.8 \\
Moderately differentiated & 28 & 45.9 \\
Poorly differentiated & 24 & 39.3 \\
Type of operation & & \\
Distal radical gastrectomy (D1) & 24 & 39.3 \\
Palliative distal radicalgastrectomy & 4 & 6.6 \\
Total radical gastrectomy & 5 & 8.2 \\
Palliative gastrojejunostomy & 9 & 14.8 \\
Feeding jejunostomy & 8 & 13.1 \\
No operation & 11 & 18.0 \\
\hline
\end{tabular}


Table IV

Comparison of clinico-pathological factors in patients positive and negative for serum CA 19-9

\begin{tabular}{|c|c|c|c|c|c|}
\hline \multirow[t]{2}{*}{ Factor } & \multicolumn{2}{|c|}{ CA $19-9+$ ve } & \multicolumn{2}{|c|}{ CA $19-9-$ ve } & \multirow[t]{2}{*}{$p$ value } \\
\hline & $\mathrm{n}$ & $\%$ & $\mathrm{n}$ & $\%$ & \\
\hline \multicolumn{6}{|l|}{$\overline{\operatorname{Sex}}$} \\
\hline Male & 12 & 85.3 & 34 & 72.3 & \multirow[t]{2}{*}{0.48} \\
\hline Female & 2 & 14.7 & 13 & 27.7 & \\
\hline \multicolumn{6}{|l|}{ Age } \\
\hline d" 55 & 10 & 71.4 & 27 & 57.4 & \multirow[t]{2}{*}{0.35} \\
\hline$>55$ & 4 & 28.6 & 20 & 42.6 & \\
\hline \multicolumn{6}{|l|}{ Histological type } \\
\hline Well Differentiated & 0 & 0.0 & 9 & 19.1 & \multirow[t]{3}{*}{0.19} \\
\hline Moderately differentiated & 7 & 50.0 & 21 & 44.7 & \\
\hline Poorly differentiated & 7 & 50.0 & 17 & 36.2 & \\
\hline \multicolumn{6}{|l|}{ Depth of invasion } \\
\hline Upto serosa(T1+T2+T3) & 3 & 25.0 & 28 & 85.0 & \multirow[t]{2}{*}{0.007} \\
\hline Beyond serosa(T4) & 9 & 75.0 & 12 & 15.0 & \\
\hline \multicolumn{6}{|l|}{ Lymph node involvement } \\
\hline Perigastric $(\mathrm{N} 0+\mathrm{N} 1)$ & 2 & 16.6 & 30 & 85.0 & \multirow[t]{2}{*}{0.000} \\
\hline Beyond (N2+N3) & 10 & 83.4 & 10 & 25.0 & \\
\hline \multicolumn{6}{|l|}{ Metastasis } \\
\hline Present & 7 & 50.0 & 8 & 17.0 & \multirow[t]{2}{*}{0.03} \\
\hline Abscent & 7 & 50.0 & 39 & 83.0 & \\
\hline
\end{tabular}

Chi- square test was done to detect significance.

Comparison of clinico-pathological factors in patients positive and negative for Serum CA 19-9 level was presented in Table IV. In 14 $(23 \%)$ patients, preoperative serum CA 19-9 levels were positive; As seen in table IV, there was no correlation with CA 19-9 level with sex $(\mathrm{P}=0.48)$ and age $(\mathrm{P}=0.35)$ of the patients. There was also no correlation with the histologic type and CA 19-9 positivity $(\mathrm{P}=0.19)$. CA 19-9 positivity had significant correlation with depth of invasion stage $(\mathrm{P}=0.007)$, lymph node involvement $(\mathrm{P}=0.000)$, with metastasis $(\mathrm{P}=0.03)$; But there was no correlation of $\mathrm{CA}$ 19.9 with stage $(\mathrm{P}=0.4)$ (Table V).

\section{Table V}

Early and advanced stages of gastric cancer confirmed after surgery based on positive or negative levels of CA 125 and CA 19.9 and their association with staging

\begin{tabular}{lcccc}
\hline & \multicolumn{4}{c}{ Number of cases } \\
\cline { 2 - 5 } Tumour marker & Serum levels & Early stages & Advanced stages & P value \\
\hline CA 19-9 & Positive & 4 & 10 & 0.4 \\
& Negative & 8 & 39 & \\
\hline
\end{tabular}

Fissure Exact test was done to detect significance. 


\section{Discussion:}

Despite progress in recent years towards the early detection of gastric cancer in the western country and Japan, most patients will already have advanced disease at diagnosis in our country. The majority of patients will die of recurrent disease, even if surgery is thought to be curative at the time. bGastric cancerspecific tumor markers have not yet been identified, and the tumor markers currently in use have very little benefit as screening tests due to their low sensitivity in early gastric cancer. ${ }^{5}$ Preoperative serum markers, especially CA 19-9, CA72-4 and CEA, have been evaluated as indicators of advanced disease and shorter prognosis and some of them have been confirmed as being significant prognostic factors, next to the three major factors of depth of invasion (T), lymph node metastasis $(\mathrm{N})$ and distant metastasis (M). Therefore, these markers are used in predicting and in monitoring patients with advanced gastric Cancer. ${ }^{8}$ It is well known that undifferentiated types of gastric Cancer seldom produce these markers. ${ }^{8}$ This study was conducted to find out the relevance of CA 19-9 and the factors age, sex, tumor infiltration, N-classification, staging, and grading in gastric carcinoma.

Same factors were studied by $\underline{\text { Reiter }}^{9}$ as classical prognostic factors to find out the relevance of CEA, CA 19-9, CA 72-4 and prognosis in gastric carcinoma. The correlation between tumor marker levels and clinicopathological features and overall survival was also studied by Duraker ${ }^{10}$. In this study, patients $<55$ years of age was $37(60.7 \%)$ and $>55$ years of age was $24(37.3 \%)$. The mean age of the patients was 52.89 years (SD, 12.11 years; range, 20-80years). Similar to our study the mean age of the patients with gastric carcinoma were 59 years ${ }^{11}$ and 58 years. ${ }^{12}$ However, in some other studies mean age was 63.6 \pm 11.7 years, ${ }^{13}$ and 61.4 years. ${ }^{14}$ In general, men are more affected by the disease than women and, as with most solid organ malignancies, the incidence increases with age. ${ }^{1}$

In this study number of male 46 (75.4\%) female $15(24.6 \%)$ ratio was $3: 1$. Similar result was found in another study in Bangladesh for gastric adenocarcinoma in which male and female ratio was 2.36:1. ${ }^{2}$ In different series, this has been reported as 29 (63.0\%) were males and $17(37.0 \%)$ females by Filho et al, ${ }^{13}$ Ucar et al 12 the male/female ratio was $1.9 / 1$ and according to Kodera et al ${ }^{14}$, with a male-tofemale ratio of 3:2.

Vomiting was presented in $41(67.2 \%)$ but it was found $75 \%$ of cases in a study by Chowdhury ${ }^{15}$. Weight loss was evident in 25 (41.0\%); weight loss seen by Sougioultzis ${ }^{16}$ was $28.9 \%$ which is similar to our study. Pain on epigastrium was presented by 39 (63.9\%) was calmost similar to that of Sougioultzis ${ }^{17}$; Malena was present in $8(13.1 \%)$ where as Chowdhury ${ }^{15}$ reported hemetemesis and melena in $6.94 \%$ cases. Anemia requiring transfusion was in $24(39.3 \%)$ in this study which was very similar to Sougioultzis ${ }^{17}$ as blood transfusion needed in $41.2 \%$ cases. History of smoking in this group was 37 (60.7\%) Smoking was $57 \%$ cases reported by Chowdhury ${ }^{15}$. Primary tumor site was the pyloric part 26 (42.6\%) body and antrum in $12(19.7 \%)$ diffuse involvement of stomach in $22(36.1 \%)$ patients. Similar involvement of antrum recorded by Sougioultzis (42.4\%). ${ }^{18}$ Those findings demarcate that most of our patients had tumors on pyloric part, most of them suffer from anaemia requiring blood transfusion. They had tendency of smoking but less alcohol intake. As most of the patients had tumors on pyloric part, hence, vomiting was more presented by our patients than dysphagia.

The tumor staging was completed according to AJCC classification of gastric carcinoma. Mos patients were at tumor at stage III $(29.5 \%)$ and 31 at stage IV (50.8\%). Most of the patients were also in stage IV in many other studies also. 12,17,19 Among differentiation well differentiated $9(14.8 \%)$, moderately differentiated 28 (45.9\%) \& poorly differentiated was $24(39.3 \%)$. In a study of our country, 357 $(57.12 \%)$ of the gastric adenocarcinomas were found poorly differentiated, 80 (12.8\%) were well differentiated and 84 (13.44\%) were moderately differentiated. ${ }^{2}$ Distal radical gastrectomy was performed in $39.3 \%$ patients, palliative distal gastrectomy in $6.6 \%$, Similar result was found 
in other studies of our country The maximum palliative surgery was distal partial gastrectomy. ${ }^{15}$ However, in our series gastric bypass surgery in $14.8 \%$ patients. In other series Gastrojejunostomy done in older group was 21 (42\%) cases Chowdhury et al. ${ }^{15}$ In other series bypass operation was done in $7.23 \%$ and $5 \%$ cases ${ }^{18}$

Among the resectable 33(54.1\%) cases, total gastrectomy was performed in 5 patients, subtotal gastrectomy in 28 , Among the non resectable cases gastric bypass surgery in 9 patients. Fanelli et $\mathrm{al}^{19}$ found that $40 \%$ of the patients underwent gastrectomy. Ucar et al. ${ }^{12}$ showed total gastrectomy was performed in 58 patients, subtotal gastrectomy in 25 , and gastric bypass surgery in 12 . Study by Hayes et al. ${ }^{18}$ showed that out of 85 patients, 13 cases were unsuitable for resection and simple bypass was performed in five of these, 11 cases with advanced disease had palliative resections.

Serum CA19-9 level was measured preoperatively in patients with gastric cancer. Although there appears to be clinical significance in measuring these markers in some studies other studies cast doubt. In the current study, we analyzed the usefulness of CA19-9 level in 61 patients with gastric cancer. A total of $14(23 \%)$ patients were seropositive for CA19-9 (range was 0.01-848.0 U/mL).

Similar to our study Wobbes $\mathrm{T}$. et $\mathrm{al}^{11}$ had the overall positivity of the tumor markers was $21 \%$ for CA 19-9. Kim DH et $\mathrm{al}^{5}$, CA 19-9 showed positivity rates of, $25-52 \%$. Basoglu $\mathrm{M}$ et al, ${ }^{20}$ serum CA 19-9 levels increased in 9 (26.3\%) patients with malignant diseases, respectively.

An elevated level were found In a study conducted by Filella et $\mathrm{al}^{21}$ serum levels CA 19.9 was measured in gastric cancer; CA 19-9 was increased in $46 \%$, By Marreli et al ${ }^{22}$ The preoperative positivity of the marker was 35\% for CA19-9. Duraker N et $\mathrm{al}^{10}$ In his series, preoperative serum CA $19-9$ positivity in gastric carcinoma was $31.5 \%$. Ucar $\mathrm{E}$ et $\mathrm{al}^{12}$ overall preoperative serum levels of CA 19-9 was $41 \%$. in S. Sougioultzis ${ }^{16}$ Ca $19-9$ level was elevated in $62.1 \%$ cases. Lower positivity found in Study of Hwang et al. ${ }^{23}$ showed Preoperative CA19-9 was above cut off level 8.7\%, of all cases respectively. Sumiya et al. ${ }^{24}$ The positivity rates of CA19-9 (e"37 U/mL) was $18 \%$. On comparison of patients positive and negative for serum CA 19-9 level and clinico-pathological Factors, there was no correlation with CA 199 and age $(\mathrm{P}=0.35)$ as in the study by Duraker ${ }^{10}$ no correlation with CA 19-9 and sex $(\mathrm{P}=0.48)$ was found in this study. However, according to Duraker ${ }^{10}$, there was significant correlation with CA 19-9 and gender (P.0.052); which didn't match with our study.

Histologic type and CA 19-9 positivity did not have any correlation in our study $(\mathrm{P}=0.19)$. Similarly Filho et al. ${ }^{13}$ and also Duraker ${ }^{10}$ the histopathologic type did not influence the positivity of the tumor markers, while Wobbes et al. ${ }^{11}$ and Cidón ${ }^{25}$ found CA $19-9$ positivity rates to be significantly higher in differentiated tumors.

The differences between reports addressing histologic type and these tumor markers may be due to the variation of histologic types in differentiated and undifferentiated tumor groups and the different rates of these histologic types in different series. The proportions of lymph node metastasis (P.000) were significantly higher in CA 19-9 positive cases. Similarly significantly higher rates of lymph node metastasis in CA 19-9 positive patients were documented in the other study. 12,14,24,25 No correlation between lymph node status and CA 19-9 level was observed in some studies. ${ }^{10,13}$ There was significant association between CA 19-9 and the depth of tumors invasion on the present study (0.007) which was similar in Duraker ${ }^{10}(0.01)$. Filho et a. ${ }^{13}$ (0.05) with carcinoma penetration into the gastric wall, Ucar et al. ${ }^{12}$ found significant difference of positive CA 19-9 level $(\mathrm{P}=0.03)$ in patients with serosal involvement. Kodera et al. ${ }^{14}$ also verified that neoplasias which extended beyond the muscle were more frequent in patients with elevated serum CA 19-9 levels. Cidón ${ }^{25}$ stated CA 19.9 positivity was closely related depth of invasion. All studies were similar to this study $(\mathrm{P}=0.04)$. Sumiya et al. ${ }^{25}$ observed that CA19-9 significantly correlated with depth of invasion. 
However, Guadagni et al. ${ }^{26}$ found no association between serosa involvement and elevated sera CA 19-9 levels. Early and advanced stages of GC confirmed after surgery based on positive or negative levels of CA 19.9 and their association with staging in Table $\mathrm{V}$ showed that when patients in stages early (I-II) and advanced (III-IV) were compared, significant difference was not observed for CA $19.9(\mathrm{P}=0.4)$. After evaluating pathological staging, we observed no significant difference in advanced stages higher tumor marker positivity rates $(P=0.4)$. Similarly other study showed that sera CA 19-9 levels in advanced neoplasias (stages III and IV) were not significantly higher $(\mathrm{P}=0.54)$ than in less advanced stages (stages I and II). Filho et al. ${ }^{13}$ and Wobbes et al. ${ }^{11}$ also did not find a correlation between disease stage and CA 19.9 positivity. However, statistically significant difference was seen between advanced and early stages with respect to CA 19-9. $10,20,25,27$

There are two major prognostic factors: depth of stomach wall tumoral invasion and involvement of RLN, which are also found significantly associated with tumor marker CA19-9 level in our study. ${ }^{25}$ Though it was not fully verified in our study, many results although controversial, suggest that both CA 19.9 and CEA levels increase proportionally with tumor burden and might identify tumor staging preoperatively. 10,14,22,25,28 However, because little research on the prognoses of gastric cancer patients with elevated preoperative CA 19-9 levels has been performed, the clinical significance of preoperative CA 19-9 levels has not been fully verified. ${ }^{29}$

\section{Conclusion:}

In conclusion, in gastric carcinoma, though preoperative serum CA 19-9 level was significantly associated with the depth of tumors invasion, lymph node involvement and metastasis but preoperative serum CA 19-9 level do not indicate the stage of the disease and does not vary with grade of the disease. Thus, the tumor marker is not helpful in deciding stage or agressiveness of gastric adenocarcinoma. As many studies suggest that Ca19-9 can identify tumor staging preoperatively so more studies with larger sample size are needed to definitively validate the marker as predictor of the pathological stages of gastric carcinoma.

\section{References:}

1. Primrose JN. Stomach and Duodenum, In: Williams NS, Bulstrode JK O'Connell PR. eds. Bailey and Love's Short Practice of Surgery. $25^{\text {th }}$ ed. London: Arnold; 2008: p.1045-79.

2. Islam SMJ, Ali SM, Ahmed S, Afroz QD, Chowdhury R, Huda M. Histopathologic pattern of gastric cancer in Bangladesh. JAFMC Bangladesh 2009; 5(1): 21-4.

3. Ilellajo X, Uster S, Olinaj RA, et al. TAG-72, CA19.9 and CEA as tumor markers in gastric cancer. Acta Oncofogica 1994; 33(7): 747-51.

4. Chan DW, Sell S. Tumor Markers. Burts CA, Ashwood ER. eds. Textbook of clinical chemistry. Philadelphia: Saunders; 1994: p.897-927.

5. Kim DH, Jong $\mathrm{S}$, Cheong $\mathrm{AH}$, et al. The relationships between perioperative CEA, CA 199, and CA 72-4 and recurrence in gastric cancer patients after curative radical gastrectomy. J Surg Oncol 2011; 104: 585-91.

6. Takahashi Y, Takeuchi T, Sakamoto J, et al. The usefulness of CEA and/or CA 19.9 in monitoring for recurrence in gastric cancer patients: a prospective clinical study. J Gastric Cancer 2003; 6: 142-5.

7. Edge SB, Byrd DR, Compton CC, et al. American Joint Committee on Cancer (AJCC) cancer staging manual. $7^{\text {th }}$ ed. New York: Springer; 2010: p. 117-21.

8. Ohkura H. Tumor markers in monitoring response to chemotherapy for patients with gastric cancer. Jpn J Clin Oncol 1999; 29(11): 525-6.

9. Reiter W, Stieber P, Reuter C, et al. Prognostic value of preoperative serum levels of CEA, CA 19-9 and CA 72-4 in gastric carcinoma. Anticancer Res 1997; 17: 2903-6.

10. Duraker N, Çelik AN. The prognostic significance of preoperative serum CA 19.9 in patients with resectable gastric carcinoma: comparison with CEA. J Surg Oncol 2001; 76: 266-71.

11. Wobbes T, Thomas CMG, Segers MFG, Nagengast FM. Evaluation of seven tumor markers (CA 50, CA 19-9 TruQuant, CA 19-9, CA 195, carcinoembryonic antigen, and tissue polypeptide antigen) in the pretreatment sera of patients with gastric carcinoma. Cancer 1992; 69: 2036-41.

12. Ucar E, Semerci E, Ustun H, et al. Prognostic value of preoperative CEA, CA 19-9, CA 72-4, and 
AFP levels in gastric cancer. Adv Ther 2008; 25: 1075-84.

13. Filho RC, Palma RT, Giusti MF, et al. Levels of carcinoembryonic antigen and CA 19-9 in the sera and peritoneal washing of patients undergoing surgical treatment for gastric carcinoma. Arq Gastroenterol 2008; 45(3).

14. Kodera Y, Yamamura Y, Shimizu Y, et al. Peritoneal washing cytology: prognostic value of positive findings in patients with gastric carcinoma undergoing a potentially curative resection. J Surg Oncol 1999; 72(2): 60-5.

15. Chowdhury SA, Hussain MM, Ahmed J. Presentation and immediate outcome of surgical treatment of patients with carcinoma of the stomach - a comparative study between young and elderly patients. J Bangladesh Coll Phys Surg 2010; 28(3): 145-50.

16. Sougioultzis S, Syrios J, Xynos ID, et al. Palliative gastrectomy and other factors affecting overall survival in stage IV gastric adenocarcinoma patients receiving chemotherapy: a retrospective analysis. Eur J Surg Oncol 2011; 37(4): 312-8.

17. Hayes N, Wayman J, Wadehra V, et al. Peritoneal cytology in the surgical evaluation of gastric carcinoma. Br J Cancer 1999; 79: 520-4.

18. Sayeed A. Management of carcinoma of stomach: problems and Strategies. J Bangladesh Coll Phys Surg 1999; 17: 54-7.

19. Fanelli MF, de Paiva TF, Jr. Silva MJB, et al. Predictors of peritoneal carcinomatosis in patients with gastric cancer treated at a single institution in Brazil. J SurgOncol 2009;100: 452-455

20. Basoglu M, Kiziltunç A, Akçay F, Gündogdu C, et al, Increased serum CA 72-4 levels in patients with gastrointestinal carcinoma. Tr J Med Sci 1998; 28: 259-63.
21. Filella X, Fuster J, Molina R, et al. TAG-72, CA 19.9 and CEA as tumor markers in gastric cancer. Acta Oncol 1994; 33(7): 747-51.

22. Marrelli D, Roviello F, De Stefano A, et al. Prognostic significance of CEA, CA 19-9 and CA 72-4 preoperative serum levels in gastric carcinoma. Oncology 1999; 57: 55-62.

23. Hwang GI, Yoo $\mathrm{CH}$, Sohn $\mathrm{BH}$, et al. Predictive value of preoperative serum CEA, CA19-9 and CA125 levels for peritoneal metastasis in patients with gastric carcinoma. Cancer Res Treat 2004; 36: $178-81$.

24. Sumiya I, Shoji N, Shuuichi H, et al. Clinical importance of preoperative Carcinoembryonic Antigen and Carbohydrate Antigen 19-9 levels in gastric cancer. J Clin Gastroenterol 2001; 32(1): 41-4.

25. Cidón EU, Bustamante R. Gastric cancer: tumor markers as predictive factors for preoperative staging. J Gastrointest Canc 2011; 42: 127-30.

26. Mattar R, Andrade CRA, Mastrantonio F, et al. Preoperative serum levels of CA 72-4, CEA, CA 19-9, and alpha-fetoprotein in patients with gastric cancer. Rev Hosp Clin Fac Med S Paulo 2002; 57: 89-92.

27. Joypaul B, Browning M, Newman E, et al. Comparison of serum CA 72-4 and CA 19-9 levels in gastric cancer patients and correlation with recurrence. Am J Surg. 1995; 169: 595-9.

28. Choi RA, Park JC, Kim JH, et al. High level of preoperative High level of preoperative carbohydrate antigen $19-9$ is a poor survival predictor in gastric cancercarbohydrate antiAntigen CA 19-9 as a poor survival predictor ingastric cancer. World J Gastroenterol 2013; 19(32): 5302-8. 